\title{
Efficacy of Infrazygomatic Crest Implants for Full-arch Distalization of Maxilla and Reduction of Gummy Smile in Class II Malocclusion
}

\author{
Amir Shaikh ${ }^{1}$, Asna Fatima Jamdar ${ }^{2}$, Shakeel Ahmed Galgali ${ }^{3}$, Smita Patil ${ }^{4}$, Inayat Patel ${ }^{5}$, Manjunath Sushilamma \\ Hemagiriyappa ${ }^{6}$
}

\begin{abstract}
Aim: The purpose of the study was to evaluate the efficacy of infrazygomatic (IZ) implants along with mini-implants for full-arch distalization of maxilla and reduction of gummy smile in patients with class II division I malocclusion.

Materials and methods: Ten orthodontic patients were taken from the department of orthodontics and dentofacial orthopedics. Each patient required distalization and intrusion of the complete maxillary arch as a part of the treatment plan. Patients were of class II malocclusion with gummy smile. Initial leveling and alignment were done by using $0.22^{\prime \prime}$ slot Mclaughlin Bennett Trevisi (MBT) prescription. Fav Anchor infrazygomatic crest (IZC) implants of $2 \mathrm{~mm}$ of head diameter and $14 \mathrm{~mm}$ length were inserted between first and second molar and $2 \mathrm{~mm}$ above the mucogingival junction in the alveolar mucosa and in the anterior region, two titanium mini-implants of $1.4 \mathrm{~mm}$ head diameter and $6 \mathrm{~mm}$ length. The screws were loaded immediately with e-chain with a minimal force from mini-implants in the anterior region to crimpable hook placed between lateral and canine and continuing the same till the IZ implants. To measure the amount of distalization and reduction of gummy smile, pre- and postlateral cephalograms were taken and assessed. Pre- and postdistalization and intrusion readings of all patients were obtained and calculated statistically for quantifying the amount of distalization of maxillary arch and intrusion for reduction of gummy smile. Results: The distalization of the maxillary arch achieved was $4.6 \mathrm{~mm}$ which is clinically and statistically significant. The anterior teeth in the study were intruded with a minimum of $3.8 \mathrm{~mm}$ which is clinically and statistically significant; the gingival smile line was reduced with a mean of $3.4 \mathrm{~mm}$ which is clinically and statistically significant. Overbite correction of $4 \mathrm{~mm}$ was done with the mean difference of $4 \mathrm{~mm}$ which is also statistically significant. Conclusion: The IZ bone screws can be effectively used as an absolute anchorage to correct class II skeletal discrepancy with gummy smile devoid of premolar extraction with noninvasive procedure.

Clinical significance: The use of IZC implants along with anterior implants, a biomechanical approach is effective in achieving full-arch distalization of maxilla and intrusion as the force vectors allow that the line of action passes through the center of resistance (Cr) of the entire maxillary arch, facilitates the distalization and intrusion of the maxillary arch, establishes ideal occlusion, and improves the smile esthetics.

Keywords: Gummy smile, Infrazygomatic crest implants, Intrusion, Maxillary arch distalization, Skeletal anchorage, Skeletal class II discrepancy. The Journal of Contemporary Dental Practice (2021): 10.5005/jp-journals-10024-3179
\end{abstract}

\section{INTRODUCTION}

Absolute anchorage through the use of one or more miniscrews has become an integral part of modern orthodontic practice.' Temporary anchorage devices (TADs) include mini-implants, miniscrews, microscrews, and micro-implants. ${ }^{2}$ The advantages of mini-implant include smaller size, greater number of implant sites, easy placement without any full flap retraction, increased patient comfort, immediate loading without any need for laboratory work, easier removal after treatment, and lower cost. ${ }^{3}$

Fayed et al. ${ }^{4}$ evaluated tomographic images of mandible and maxilla to define safe zones for placing mini-implants. In the maxilla, the optimal sites of insertion of mini-implant placement in the anterior region are between central and lateral incisors and between lateral incisor and canine in mandible. At the buccal aspect of the posterior region of both the jaws, the optimal sites are between the second premolar and first molar and between first molar and second molar.

The TADs, when placed in the inter-radicular area, pose a risk of injuring the root while inserting a mini-implant in the dentoalveolar area. It can also impede tooth movement, when the moved tooth approximates mini-implant. Therefore, the best way to prevent root injury is to place TADs in the extra-alveolar site. ${ }^{5}$
${ }^{1-5}$ Department of Orthodontics and Dentofacial Orthopedics, Al-Ameen Dental College and Hospital, Vijaypura, Karnataka, India

${ }^{6}$ Department of Periodontology, SMBT Dental College and Hospital, Sangamner, Maharashtra, India

Corresponding Author: Amir Shaikh, Department of Orthodontics and Dentofacial Orthopedics, Al-Ameen Dental College and Hospital, Vijaypura, Karnataka, India, Phone: +91 9422613939, e-mail: amirshaikhortho@gmail.com

How to cite this article: Shaikh A, Jamdar AF, Galgali SA, et al. Efficacy of Infrazygomatic Crest Implants for Full-arch Distalization of Maxilla and Reduction of Gummy Smile in Class II Malocclusion. J Contemp Dent Pract 2021;22(10):1135-1143.

Source of support: Nil

Conflict of interest: None

The treatment alternatives for adult skeletal class II division I with gummy smile patients are camouflage treatment and surgical correction. In camouflage treatment, the premolars are extracted to solve crowding, retract the incisors, and provide class I canine relationship.

Maxillary arch distalization is a favored option to correct the class II malocclusion with nonextraction approach. Headgear had 
been the conventional method for class II malocclusion through distalization of molars or entire the maxillary dentition. However, the main disadvantage is the patient compliance.

To overcome unwanted side effects, recent techniques such as maxillary arch distalization by mini-implants are more effective and give good results.

The IZC has been an obvious choice for miniscrew insertion because of the thickness of the cortical plate and its distance from the dental arch. Its ability to be placed high up in the maxillary sulcus, i.e., in the infrazygomatic (IZ) area, allows unobstructed tooth movement during retraction. The flexibility of allowing higher placement in the maxillary region allows both vertical vectors of force for intrusion mechanics.

There are many studies available in the literature regarding reducing the gummy smile with mini-implants and full-arch distalization of maxillary arch but there are very few studies in which mini-implants are used for simultaneous intrusion and full-arch distalization. Various companies Bio-Ray, Fav Anchor, etc., are promoting their IZC screws to correct the above malocclusion.

Among various intrusion mechanics applied during orthodontic treatment, the application of IZC implants is known for its effectiveness in full-arch intrusion along with distalization in maxillary dentition and help in reduction of gummy smile.

This has created an interest to undertake the study to evaluate the effectiveness of IZC implants along with mini-implants in full-arch distalization of maxilla and reduction of gummy smile in class II division I patients.

The study was designed to evaluate the efficacy of IZC implants along with mini-implants clinically and cephalometrically for full-arch distalization of maxilla and reduction of gummy smile in patients with Angle's class II division I malocclusion with the following objectives:

- To determine the full-arch distalization of the maxilla with IZC implants (Fav Anchor),

- To determine the reduction of gummy smile in the maxillary arch with IZ implants,

- To compare pretreatment and postfull-arch distalization and intrusion of maxillary arch cephalometrically.

\section{Materials and Methods}

\section{Methodology}

The present study was a prospective cephalometric evaluation study. It involved 10 patients reporting to the Department of Orthodontics and Dentofacial Orthopedics for orthodontic treatment at Al-Ameen
Dental College and Hospital, Vijayapura, Karnataka. The anticipated mean difference of pre- and postoperative study parameter as 5.56 and anticipated SD at baseline and end line as 3.1 and 2.4 and the study with $90 \%$ power and $5 \%$ level of significance was taken.

Case selection for the study was done based on the following criteria: (1) patients with a mean age of 15-30 years, (2) patients with Angle's class II division I malocclusion, (3) gummy smile $-2 \mathrm{~mm}$ or more maxillary gingiva shown on full smile, (4) deep over bite, and (5) good patient compliance during the treatment with good oral hygiene.

The subjects with the following conditions were excluded from the study: (1) patients indicated for extraction treatment, (2) The subjects who had more than $3 \mathrm{~mm}$ incision-stomium distance, (3) patients showing symptoms of temporomandibular disorders, and (4) patients with periodontal diseases.

Initially, all the essential orthodontics diagnostic aids, i.e. orthopantomograph (OPG), lateral cephalogram, study models, intraoral and extraoral photographs, were taken. After the preorthodontic treatment, informed consent of the patient for placement of IZ screws and mini-implants was taken.

Orthodontic treatment carried out with 0.22 slot MBT prescription after initial leveling and aligning with 0.16 HANT, $19 \times 25$ HANT, and $19 \times 25$ SS.

Lidayn surface anesthetic $100 \mathrm{~g}$ was sprayed at the implant insertion site. Lignox $2 \% \mathrm{~A}$ was administered near the miniimplant sites. The two posterior IZ screws (Fav Anchor, size $2 \mathrm{~mm} \times 14 \mathrm{~mm}$ ) were inserted between first and second molar and $2 \mathrm{~mm}$ above the mucogingival junction in the alveolar mucosa that was directed at $90^{\circ}$ to the occlusal plane (OP). After the initial notch in the bone, the bone screwdriver direction was changed by 55 to $70^{\circ}$ toward the teeth and driving the screw to the IZ area of the maxilla. The bone screw was screwed in till only the head of the screw was visible outside the alveolar mucosa (Fig. 1).

Two anterior titanium alloy miniscrews of $6 \mathrm{~mm}$ length (Fav Anchor; size $1.4 \times 6 \mathrm{~mm}$ ) were inserted between incisors and canines (Fig. 2A). Panoramic radiographs were taken to confirm the position of the mini-implants (Fig. 2B).

The screws were loaded immediately with elastomeric chain with a minimal force from mini-implants in the anterior region to crimpable hook placed between lateral and canine and continuing the same till the IZ implants. The patients were recalled every 4 weeks, and the screws were checked for signs of mobility or infection. The distalizing force was continued till class I molar and canine relation is attained (Fig. 3).
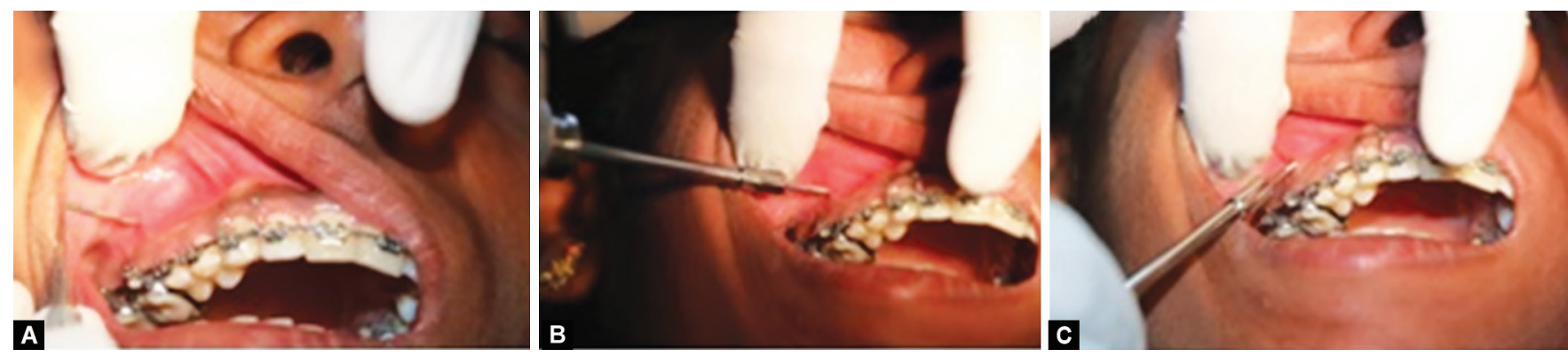

Figs $1 \mathrm{~A}$ to $\mathrm{C}$ : The bone screw was screwed in till only the head of the screw was visible outside the alveolar mucosa 

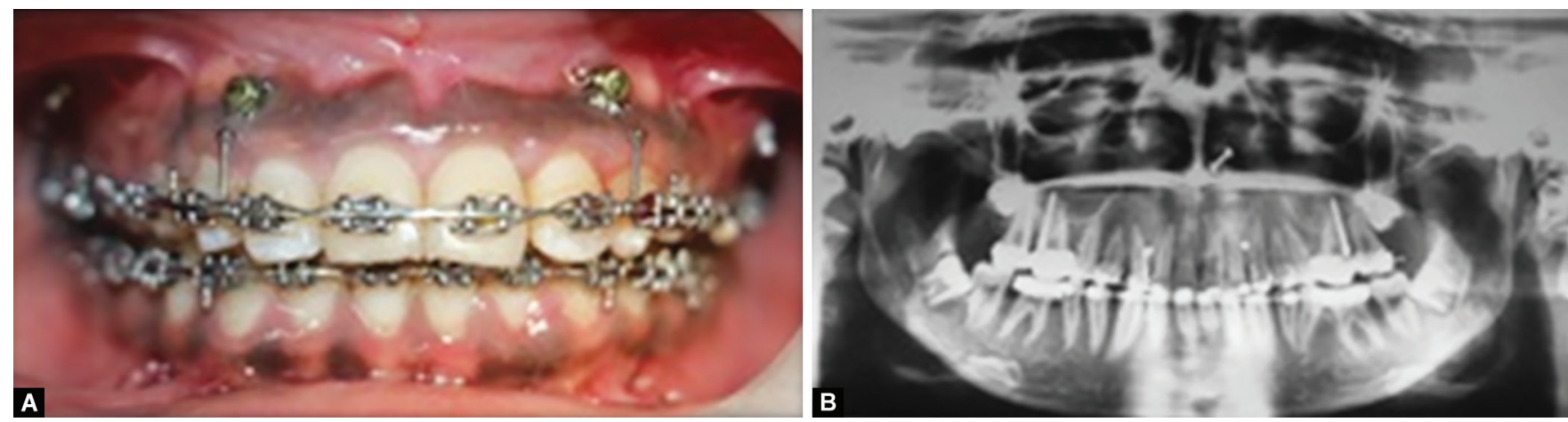

Figs 2A and B: (A) Miniscrews of $6 \mathrm{~mm}$ length (Fav Anchor; size $1.4 \times 6 \mathrm{~mm}$ ) were inserted between incisors and canines; (B) Panoramic radiographs were taken to confirm the position of the mini-implant
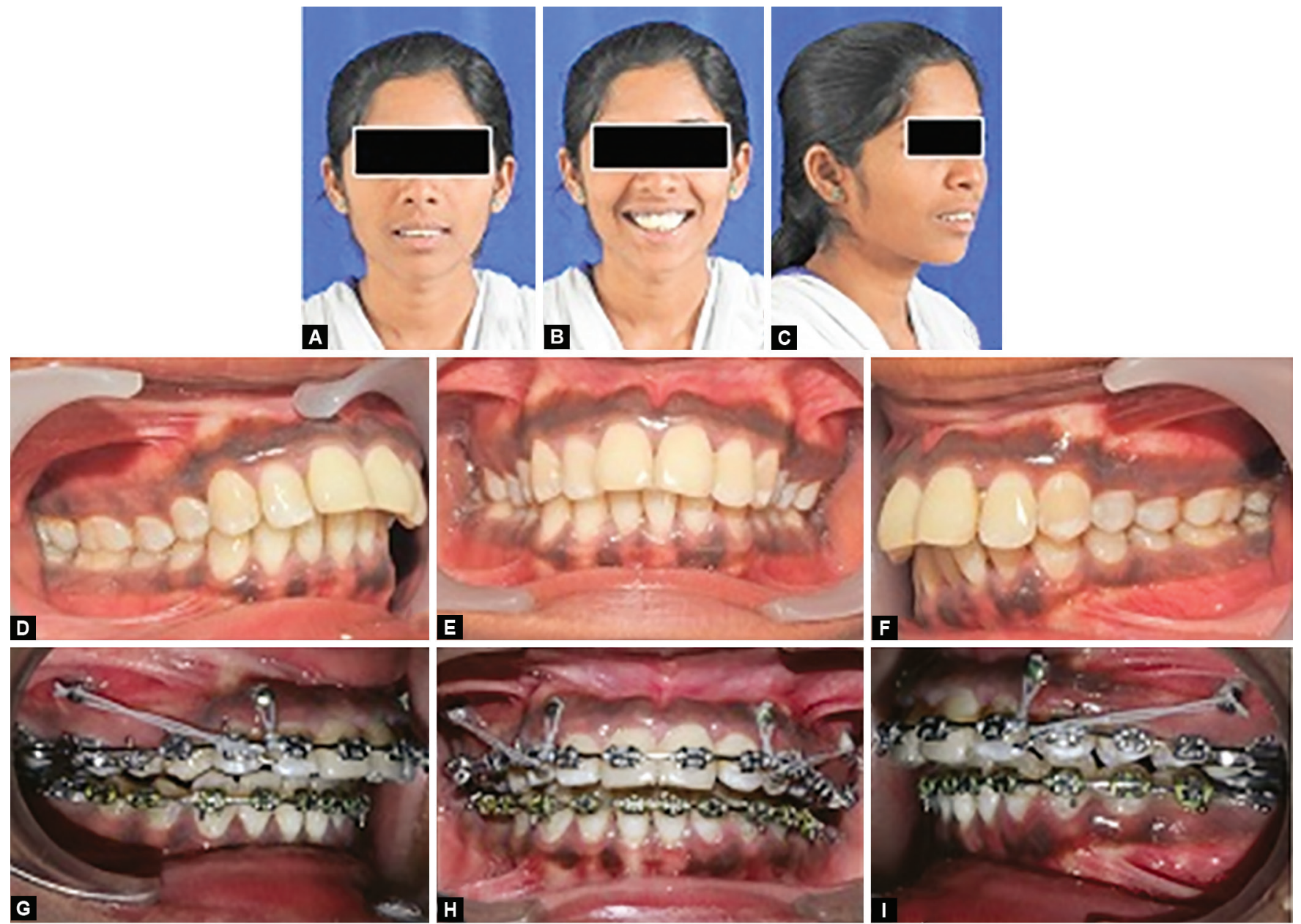

Figs 3A to I: Predistalization and intrusion-Extraoral photographs: (A) Facial profile; (B) Facial profile with smile; and (C) Lateral profile. Intraoral photographs: (D) Right view; (E) Frontal view; and (F) Left view. After placement of IZC implants and mini-implants; (G) Right; (H) Frontal; and (I) lateral view

\section{Cephalometric Parameters Distalization of Full Maxillary Arch}

Two reference lines were constructed for horizontal and vertical measurements. In the pre- and posttreatment lateral cephalogram, a line parallel to the initial OP was drawn through the anterior nasal spine (ANS), which was named the horizontal reference (HR); a line perpendicular to HR was drawn through Sella as the vertical reference (VR) line (Fig. 4A). Distal movement was measured from the following:

- VR-edge of U1.

- VR-distal cusp tip U6. 

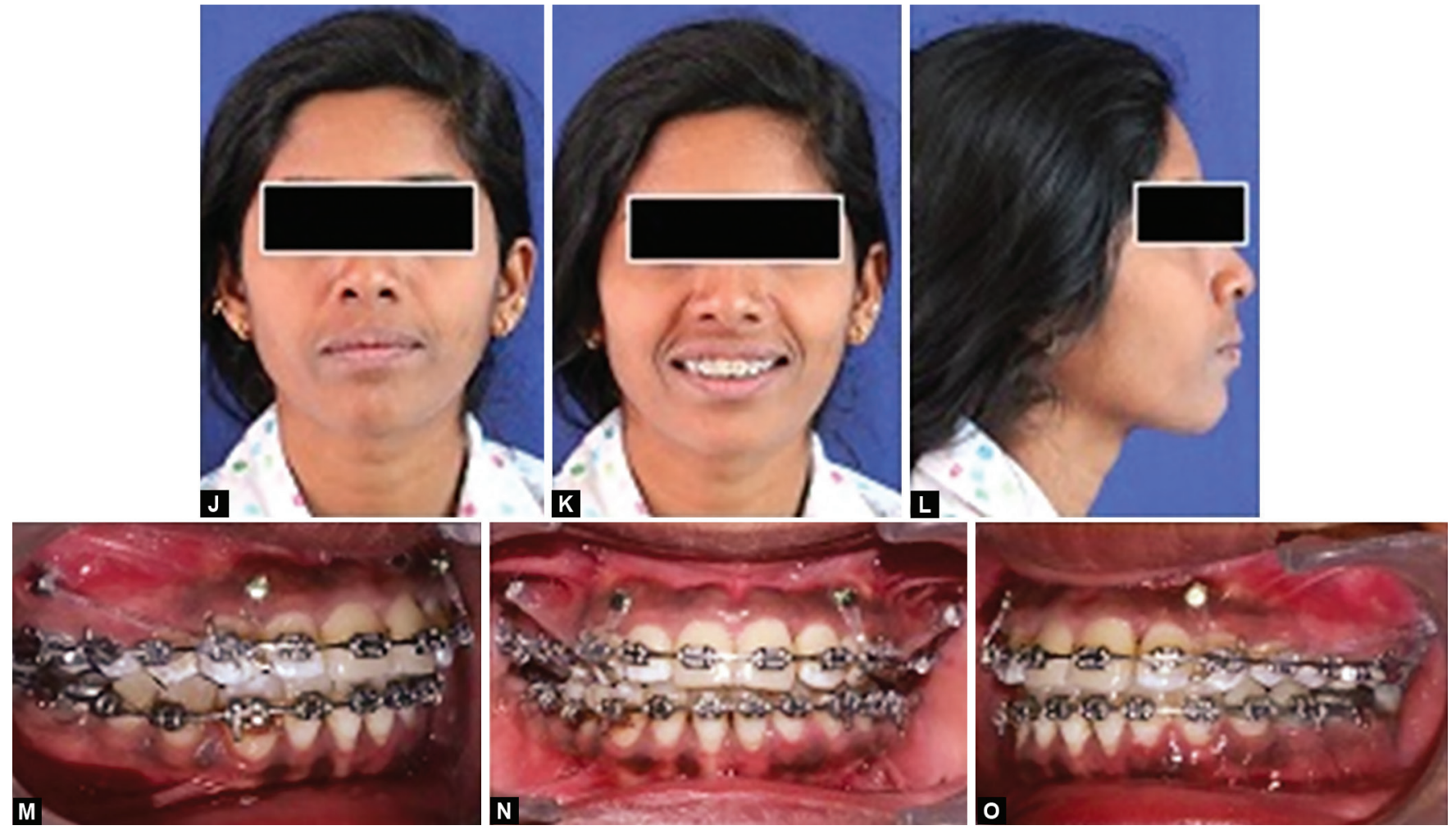

Figs 3J to O: Postdistalization and intrusion-Extraoral photographs: (J) Facial profile; (K) Facial profile with smile; and (L) Lateral profile. Intraoral photographs: (M) Right view; (N) Frontal view; and (O) Left view

- Pterygomaxillary fissure (Ptm) vertical line is drawn perpendicular to the Frankfort horizontal plane.

The distalization of maxillary arch is measured from the following:

- distal cusp of U6 to Ptm vertical.

- Point A-Ptm vertical.

\section{Cephalometric Parameters for Gummy Smile Reduction}

The following variables were measured to assess the gummy smile reduction illustrated in Figure 4B:

- Incision to stomion in millimeters (In-Sto).

- Incision to palatal plane in millimeters (U1-PP).

- Incisal apex to palatal plane in millimeters (U1apex-PP).

- Upper molar to palatal plane millimeters (U6-PP).

- Lower molar to mandibular plane in millimeters (L6-MP).

- Lower facial height to total facial height ratio (LFH/TFH).

- Upper incisors to FH (U1-FH) angle.

- HR-U1.

\section{Method for Determination of Full Distalization of Maxillary Arch and Reduction of Gummy Smile}

Lateral cephalograms were taken before (T0) and after (T1) maxillary arch distalization using the Vatech machine (Fig. 5). The radiographic magnification of the cephalograms was standardized as $100 \%$ of the actual size, with a100-mm metal ruler image captured in the digital film as a reference.

Assessments of the soft tissue skeletal and dental changes were done by tracing the pre- and postdistalization and intrusion lateral cepahlographs by using the above-mentioned parameters.
Data were analyzed using SPSS software v.23 (IBM Statistics, Chicago, USA) and Microsoft Office 2007. All characteristics were summarized descriptively. For continuous variables, the summary statistics of mean \pm standard deviation (SD) were used. The difference of the means of analysis variables between two time points in same group was tested by paired $t$ test. If the $p$-value was $<0.05$, then the results were considered to be statistically significant otherwise it was considered as not statistically insignificant. ${ }^{6-24}$

\section{Results}

A total of 11 parameters have been taken for the assessment of distalization of the maxillary arch.

SNA and SNB angle observed from pre- and postdistalization was statistically nonsignificant with $p=0.053$ which indicate that the anteroposterior position of the maxilla with regard to cranial base is not significantly changed. SN-U1 was found to be significantly decreased from $110^{\circ}$ to $102^{\circ}$ which showed statistically significant with $p<0.001$, showed the reduction in proclination of incisors. Upper incisor inclination was reduced by $9^{\circ}$ (Table 1 and Fig. 6).

The dental linear measurements PTV-U1and PTV-U6 were found to be significantly changed indicating distalization of the maxillary arch. Retraction of the upper incisors and distalization of upper first molar was significant statistically, $p<0.001$. Incisor retraction of $5 \mathrm{~mm}$ was seen. Distalization of the maxillary first molar was observed as $4.6 \mathrm{~mm}$. There was a significant improvement in point A position as measured by PTV-point A where $p=0.001$ as shown in Table 1 and Fig. 6.

Statistically significant distal movement was achieved in parameters VR-U1, VR-U6 with $p<0.001$ indicating clinically 

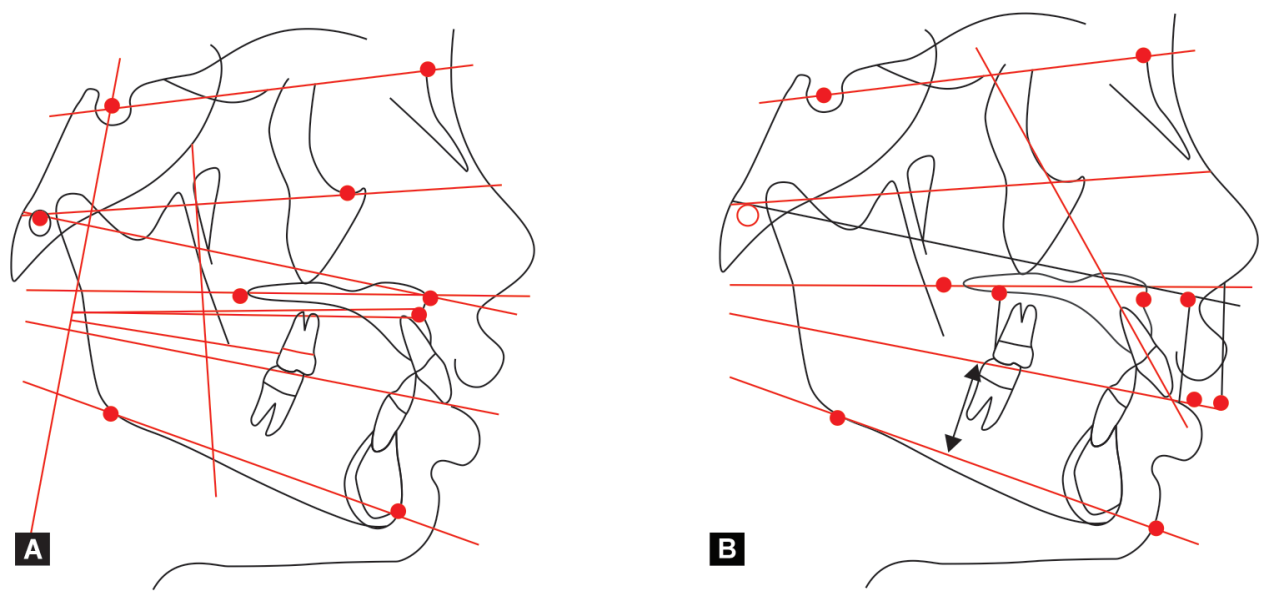

Figs 4A and B: (A) Lateral cephalogram parameters taken to measure distalization of the maxillary arch; (B) Lateral cephalogram parameters taken to measure gummy smile reduction
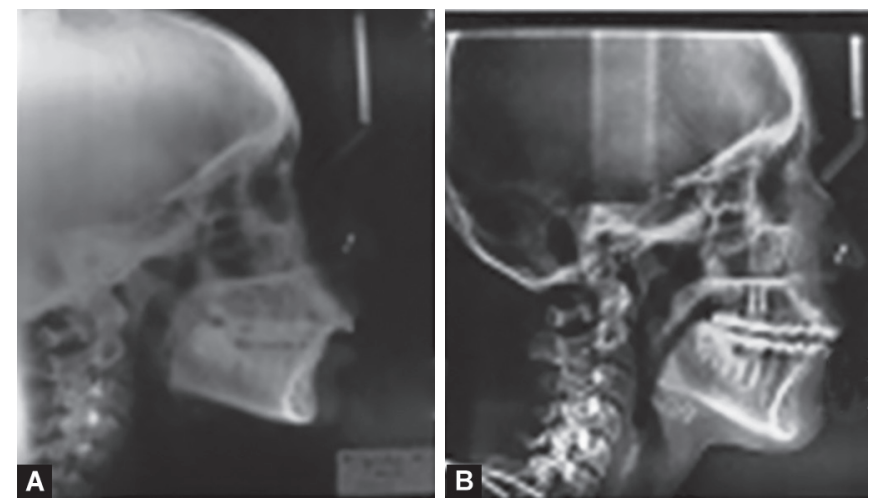

Figs 5A and B: (A) Predistalization and intrusion lateral cephalographs; (B) Postdistalization and intrusion lateral cephalographs

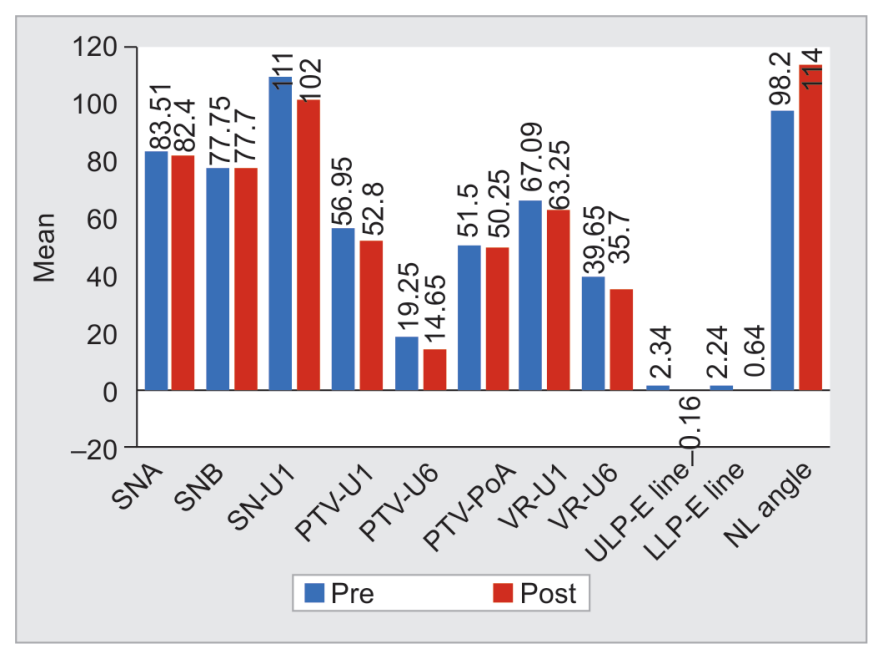

Fig. 6: Comparison of pre- and postdistalization of the complete maxillary arch

meaningful total arch distalization. Retraction observed from pre-and postdistalization upper lip to $E$ line $(2.1 \mathrm{~mm})$ was statistically significant with $p<0.001$ and lower lip-E line $(1.6 \mathrm{~mm})$ was statistically significant with $p=0.001$. There was a significant improvement in the nasolabial angle with mean value from $98.2^{\circ}$ to $114.2^{\circ}$ indicating a significant increase in the angle and was statistically significant with $p<0.001$ (Table 1 and Fig. 6).

\section{Intrusion of the Maxillary Anterior Teeth}

A total of seven parameters have been taken to determine the intrusion of the maxillary teeth. The vertical dental parameters HR-U1 and U1-PP showed significant intrusive displacement of the incisor tip. The difference HR-U1 from mean value 33.6 to $30.4 \mathrm{~mm}$ was $3.2 \mathrm{~mm}$ (SD \pm 1.3 ) which was statistically significant with $p=0.001$, and the difference of U1-PP from the mean value of 33.9-30.1 $\mathrm{mm}$ was $3.8 \mathrm{~mm}(\mathrm{SD} \pm 0.9)$ statistically significant with $p<0.001$.

PP-U6 observed from pre- and postintrusion showed statistically insignificant with $p=0.070$. The angular parameter U1-FH showed significant change indicating the reduction of the proclination of the anterior teeth and is statistically significant with $p<0.001$.

The parameters L6-MP indicating lower posterior dental height were statistically insignificant with $p=0.078$. The vertical parameter in-sto the pre- and postintrusion was found to be significantly reduced from the mean value of 5.8-2.5 $\mathrm{mm}$ with a difference of $3.3 \mathrm{~mm}$ was statistically significant with $p<0.001$.

LFH/TFH showed statistically insignificant with $p=0.260$ (Table 2 and Fig. 7).

\section{Gingival Smile Line and Overbite}

All the patients in the study showed improvement in the smile, which was assessed by using two parameters. Pretreatment mean value of the gingival smile line was $4 \mathrm{~mm}$, whereas the mean value of the postintrusion of gingival smile line was $0.6 \mathrm{~mm}$. The amount of reduction of gingival smile line was $3.4 \mathrm{~mm}$ which was statistically significant at $p<0.001$ level.

A perceptible reduction of deep bite was achieved which is statistically significant $(p<0.001)$ from premean value of $6.4 \mathrm{~mm}$ to postmean value of $2.4 \mathrm{~mm}$ with a change of $4 \mathrm{~mm}$ (Table 3 and Fig. 8).

Distalization of complete maxillary arch was attained, and the maxillary anterior teeth were intruded which significantly reduced the gummy smile with the use of IZC implants and anterior miniimplants. Distalization of maxillary arch achieved was $4.6 \mathrm{~mm}$ which is clinically and cephalometrically significant. The anterior 
Infrazygomatic Crest Implants and Reduction of Gummy Smile

Table 1: Comparison of pre- and postdistalization of the complete maxillary arch

\begin{tabular}{|c|c|c|c|c|c|c|c|c|}
\hline \multirow{2}{*}{$\begin{array}{l}\text { Parameters for distalization } \\
\text { of maxillary arch }\end{array}$} & \multicolumn{2}{|c|}{ Pre } & \multicolumn{2}{|c|}{ Post } & \multirow[b]{2}{*}{$t$} & \multirow[b]{2}{*}{ Df } & \multirow[b]{2}{*}{$p$ value } & \multirow[b]{2}{*}{ Remarks } \\
\hline & Mean & $S D$ & Mean & $S D$ & & & & \\
\hline SNA & 83.5 & 3.2 & 82.4 & 3.8 & 2.113 & 9 & 0.053 & NS \\
\hline SNB & 77.8 & 2.4 & 77.7 & 2.5 & 0 & 9 & 0.999 & NS \\
\hline SN-U1 & 111.0 & 6.3 & 102.0 & 1.8 & 5.4 & 9 & $<0.001^{*}$ & $S$ \\
\hline PTV-U1 & 57.0 & 6.2 & 52.8 & 5.0 & 5.681 & 9 & $<0.001^{*}$ & $S$ \\
\hline PTV-U6 & 19.3 & 3.6 & 14.7 & 3.6 & 20.804 & 9 & $<0.001^{*}$ & $S$ \\
\hline PTV-point A & 51.5 & 3.8 & 50.3 & 3.7 & 11.18 & 9 & $<0.001^{*}$ & S \\
\hline VR-U1 & 67.1 & 8.4 & 63.3 & 8.5 & 12.958 & 9 & $<0.001^{*}$ & $S$ \\
\hline VR-U6 & 39.7 & 4.5 & 35.7 & 4.6 & 15.019 & 9 & $<0.001^{*}$ & $S$ \\
\hline ULP-E LINE & 2.3 & 1.8 & -0.2 & 2.3 & 6.128 & 9 & $<0.001^{*}$ & S \\
\hline LLP-E LINE & 2.2 & 2.8 & 0.6 & 2.3 & 4.581 & 9 & $0.001^{*}$ & S \\
\hline NL angle & 98.2 & 11.9 & 114.2 & 11.0 & -5.795 & 9 & $<0.001^{*}$ & S \\
\hline
\end{tabular}

"Significant at $5 \%$ level of significance $(p<0.05)$

Table 2: Comparison between pre- and postintrusion of maxillary anterior teeth

\begin{tabular}{|c|c|c|c|c|c|c|c|c|}
\hline \multirow{2}{*}{$\begin{array}{l}\text { Parameters for intrusion } \\
\text { of maxillary anterior teeth }\end{array}$} & \multicolumn{2}{|c|}{ Pre } & \multicolumn{2}{|c|}{ Post } & \multirow[b]{2}{*}{$t$} & \multirow[b]{2}{*}{$d f$} & \multirow[b]{2}{*}{$p$ value } & \multirow[b]{2}{*}{ Remarks } \\
\hline & Mean & $S D$ & Mean & $S D$ & & & & \\
\hline HR-UI & 33.6 & 4.6 & 30.4 & 3.3 & 4.951 & 9 & $0.001^{*}$ & $\mathrm{~S}$ \\
\hline U1-PP & 33.9 & 4.9 & 30.1 & 4.0 & 7.926 & 9 & $<0.001^{*}$ & $S$ \\
\hline U6-PP & 27.9 & 4.4 & 27.3 & 4.1 & 2.059 & 9 & 0.070 & NS \\
\hline U1-FH & 118.9 & 4.1 & 113.1 & 4.2 & 18.673 & 9 & $<0.001^{*}$ & $S$ \\
\hline L6-MP & 34.2 & 6.2 & 33.3 & 5.6 & 2.456 & 9 & 0.078 & NS \\
\hline IN-STO & 5.8 & 0.8 & 2.5 & 0.5 & 13.102 & 9 & $<0.001^{*}$ & $S$ \\
\hline LFH/TFH & 63.8 & 1.4 & 63.7 & 1.3 & -1.203 & 9 & 0.260 & NS \\
\hline
\end{tabular}

${ }^{*}$ Significant at $5 \%$ level of significance $(p<0.05)$

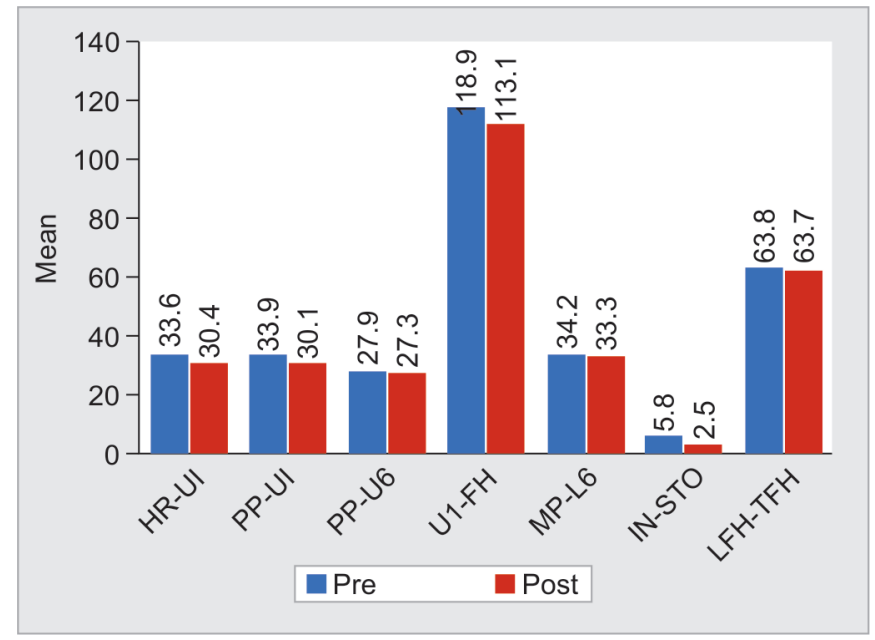

Fig. 7: Comparison between pre- and postintrusion of maxillary anterior teeth

teeth were intruded with a minimum of $3.8 \mathrm{~mm}$, and the gingival smile line was reduced with a mean of $3.4 \mathrm{~mm}$ and a mean of $4-\mathrm{mm}$ overbite correction was done. The maxillary anterior teeth were retracted with a mean of $5 \mathrm{~mm}$ which is clinically and statistically significant. There was a great improvement in nasolabial angle with $12^{\circ}$ change.

\section{Discussion}

Anchorage has been a daunting factor for many years in orthodontics. Anchorage is the salient factor that affects the treatment plan and outcome of the treatment. Many modalities of anchorage have been suggested like extra-oral anchorage, use of opposing anchors, increasing number of teeth in anchor units that have unwanted side effects and require patient compliance, but the extra-oral anchorage is difficult to use and causes injury that effects the patient compliance to use it.

Treatment of class II skeletal discrepancy patients is challenging to the orthodontists. But with the emergence of TADs which were used as skeletal anchorage. The advent of orthodontic bone screws has widened the horizon of orthodontic treatment where the borderline surgical cases and extraction cases can now be treated nonsurgically and with a nonextraction approach.

In the present study, the entire maxillary arch was distalized; at the same time, maxillary anterior teeth were intruded. Before the invention of IZC, mini-implants were used to distalize the maxillary arch and intrude the maxillary anterior teeth, but the results were not so satisfactory; hence, the study was undertaken to use IZC along with mini-implants to distalize the maxillary arch and intrude upper anterior teeth also reducing the gingival smile line and overbite. Few factors related to this study needs to be discussed.

The treatment for patients with class II division I malocclusion with gummy smile and mild skeletal mandibular deficiency can be camouflaged by the distalization of the entire maxillary arch. 
Table 3: Comparison between pre- and postgingival smile line and overbite

\begin{tabular}{|c|c|c|c|c|c|c|c|}
\hline \multirow{2}{*}{$\begin{array}{l}\text { Clinical } \\
\text { parameters }\end{array}$} & \multicolumn{2}{|c|}{ Pre } & \multicolumn{2}{|c|}{ Post } & & \multirow[b]{2}{*}{ Df } & \multirow[b]{2}{*}{$p$ value } \\
\hline & Mean & $S D$ & Mean & $S D$ & & & \\
\hline $\begin{array}{l}\text { Gingival } \\
\text { smile line }\end{array}$ & 4.0 & 1.0 & 0.6 & 0.7 & 20.821 & 9 & $<0.001^{*}$ \\
\hline Overbite & 6.4 & 2.0 & 2.4 & 0.5 & 7.667 & 9 & $<0.001^{*}$ \\
\hline
\end{tabular}

"Significant at $5 \%$ level of significance $(p<0.05)$

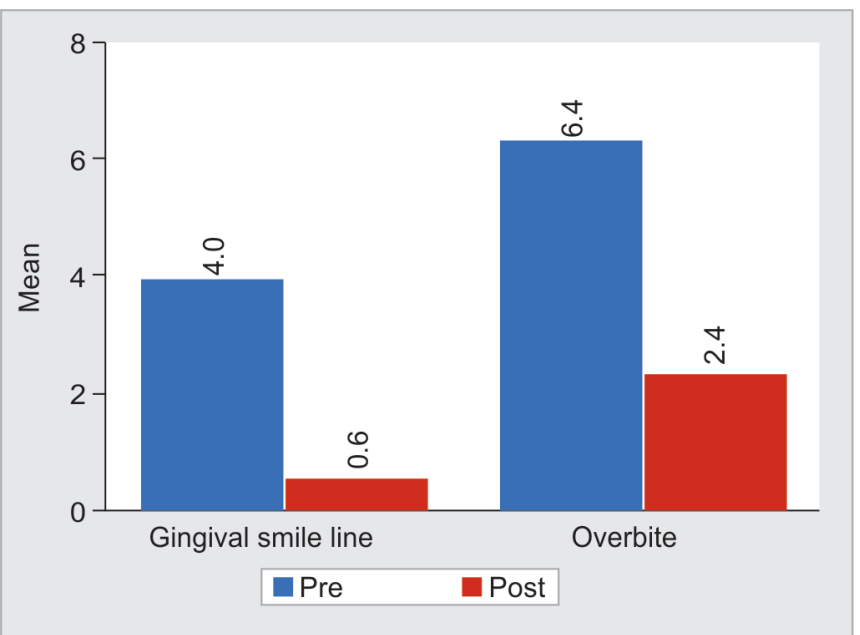

Fig. 8: Comparison between pre- and postgingival smile line and overbite

In the conventional biomechanical concepts, where a segment of teeth has been the target of orthodontic movement, the advanced technology allows the predictable displacement of the whole arch, that is, in turn may be dictated by the direct relationship between the center of resistance $(\mathrm{Cr})$ of whole arch and the line of action generated between the miniscrew and force application points at the arch wire. $\mathrm{Cr}$ of entire dental arch was localized at the mesial side of the second premolar in the maxillary arch and at the interradicular area between the second premolar and the first molar in the mandibular arch, respectively.

For instance, a rotational movement will result as the line of force passes far from the $\mathrm{Cr}$, which a force vector that runs through the $\mathrm{Cr}$ would lead to translation of the dental arch in the horizontal and/or vertical direction.

Bechtold et al. ${ }^{25}$ found an intrusion of the whole dental arch using dual inter-radicular miniscrews in the maxilla. Additional force vector created by inter-radicular miniscrew positioned between the first and second premolars changed the relationship between the imaginary $\mathrm{Cr}$ of the whole arch and the line of force, leading to simultaneous upward and backward displacement of the entire dental arch.

Sanap et al. ${ }^{26}$ compared the amount of maxillary arch distalization and its effects, on adjacent teeth, by varying the positions of mini-implants. Maximum amount of maxillary arch distalization was seen when IZ screws placed between upper first and second molar, whereas the maximum amount of maxillary arch intrusion and less distalization was observed when miniscrews placed between upper first premolar and second premolar and in between second premolar and upper first molar.
Liou et al. ${ }^{27}$ utilized CBCT to define the position of IZC screws relative to the upper molars, determined the screw positions that are detrimental for full-arch retraction, and developed new techniques to improve the success rate for retracting buccal segments and suggested that IZC 7 site is an easier and safer location for screw placement because the buccal bone plate is thicker; compared to the IZC 6 site, a $2 \times 8 \mathrm{~mm}$ SS screw in the IZC 7 site, with the platform in close contact with the gingiva, is adequate anchorage for retracting the entire maxillary arch. The shorter screw rarely interferes with the molar roots.

The thickness of the cortical bone is of vital importance for stability of mini-implants placed, and one of the common reasons for the failure of mini-implant is insufficient bone. The implant stability here depends on mechanical interdigitation of bone and implant rather than osseointegration. Therefore, bone quantity seems to be the major factor in the stability of miniscrews.

Stability of miniscrew implants depends on the quality (bone density) and quantity (bone volume) of the cortical bone. The main objective of an orthodontic screw is to gain maximum retention by placing the screw in an area with the thinnest soft tissue and the thickest cortical bone. Various anatomical sites for the placement of miniscrews have been discussed by Kim et al., ${ }^{28}$ who conducted CBCT study to provide safe locations for clinicians to place mini-implants where they observed that the inter-radicular space between the second premolar and first molar root in the upper arch and inter-radicular space between the first molar and the second molar root in the lower arch were suitable sites. Kuroda et al. ${ }^{29}$ stated that the major problem encountered with placing TADs inter-radicularly is the increased risk of root approximation that hinders the tooth movement, before the tooth moved to the expected position and is already touching the TAD. This situation can be prevented by placing implants in the extra-alveolar region.

The extra-alveolar sites available for placement of TADs include incisive fossa, premaxillary region, midpalatal region, and symphysis, canine fossa, IZC, anterior external oblique ridge (AEOR), retromolar area, and sublingual fossa.

Of all extra-alveolar sites, our study of interest is the $\mathrm{IZ}$ region. The IZC is an extra-alveolar placement site in the maxilla for orthodontic miniscrews or miniplates. It has been successfully used to provide skeletal anchorage for maxillary canine retraction, anterior retraction, en-masse anterior retraction, and intrusion of the maxillary posterior teeth.

Anatomically, the IZ crest is a thick pillar of cortical bone along the zygomatic process of the maxilla. Clinically, it is a palpable bony ridge running along the curvature between the alveolar and zygomatic processes of the maxilla. Studies of Kanomi and Schnelle concluded that it is between the maxillary second premolar and first molar, in younger subjects and is above the maxillary first molar in adults. It is recommended to insert miniscrews at a height beyond the root apex, where interseptal bone is thicker and there is a less chance of root injury.

Liou et al. In their study stated that the IZ crest above the maxillary first molar was significantly thicker than the lateral wall of the maxillary sinus. The IZ crest is usually used as an insertion site for orthodontic skeletal anchorage, because of its thicker bone. They also stated that the IZ crest has two cortical plates-the buccal cortical plate and the sinus floor. This is anatomically advantageous as it allows for bicortical fixation and also offers a better shot at primary stability of the miniscrew. 
With advanced technology, excessive gingival display during smiling treated by intrusion of the anterior segment with miniimplants. True intrusion takes place when the forces are directed through $\mathrm{Cr}$. If the intrusion force is applied anteriorly to the $\mathrm{Cr}$ of a unit, flaring occurs. There is no chance of incisor flaring if implants are placed bilaterally between the lateral incisors and the canine as they were in the study; the Cr of six maxillary anterior teeth is estimated to be half way between the $\mathrm{Cr}$ of four incisors and that of canine.

Saxena et al. ${ }^{30}$ evaluated the efficacy of mini-implants as intraoral anchorage during en-masse intrusion of six maxillary anterior teeth. Patients were undergone treatment who required true intrusion. After alignment mini-implants were placed bilaterally between the lateral incisor and canine, an intrusive force of $45 \mathrm{CN}$ given with e-chain bilaterally patient records taken at beginning and end of intrusion. Results showed statistical and clinically significant intrusion.

In the present study, IZC implants for full-arch distalization to correct class II malocclusion have been used. IZC miniscrews were used in accordance with the studies conducted by Lin and Lee to ensure bicortical engagement and 10-12 Oz (284-340 g) of distalizing force; this present study used $300 \mathrm{~g}$ of force for distalization with e-chain from the mini-implant to reverse power arm placed between lateral incisor and canine.

Almeida ${ }^{31}$ said that mini-implants in the IZC generate a retraction force system during distalization of the entire maxillary arch. The retraction force in the entire maxillary arch generates intrusive force in the molars and extrusive force in the incisors, caused by clockwise rotation around the $\mathrm{Cr}$ of the entire maxilla, which is located between the premolars. The force line of action passes below (occlusal) the maxillary $\mathrm{Cr}$ and, consequently, causes this rotation. For that reason, incisor extrusion must be expected, which may be unfavorable for patients with deep bite and gummy smile. On the contrary, this OP clockwise rotation favors simultaneous open bite closure and class II correction.

So, in our study, patients with deep bite and gingival smile, anterior intrusion with inter-radicular mini-implants, and miniimplants in IZC would be an option. In order to balance the clockwise rotation effect of the maxillary OP and favor gingival smile correction, while promoting anchorage to anterior retraction, it was suggested that aside from two IZC mini-implants, another two were to be installed between lateral incisor and canine. The oblique component of force resultant caused maxillary intrusion and the vertical component of force causes distalization of the maxillary arch.

There was a significant decrease in PTV-point A with a nonsignificant increase in point $B$ that depicts the correction of dentoalveolar class II pattern. This was due to the retraction of maxillary incisors leading to the remodeling of subspinale area.

In the study although the full dentition of the maxilla was distalized, the intrusion of the posterior teeth prevented the wedging effect; thus, the lower LFH change was statistically insignificant. Hence, it was inferred unlike conventional distalization mechanics that are only suitable for hypodivergent faces, IZC distalization can be used also for hyperdivergent patients.

There was a significant change in pterygomaxillary vertical Ptmv-U1, Ptmv-U6, VR-U1, VR-U6 which indicate the complete distalization of maxillary dentoalveolar component which was statistically significant. The distalization of the maxillary molars achieved was $4.6 \mathrm{~mm}$.

Parameters ANS-PNS (PP)-U1 and VR-U1 were used to determine the amount of intrusion. The anterior teeth in the study were intruded with a minimum of $3.8 \mathrm{~mm}$ which is clinically and statistically significant. The gingival smile line was reduced with a mean of $3.4 \mathrm{~mm}$ which is clinically and statistically significant. Overbite correction $4 \mathrm{~mm}$ was done with the mean difference of $4 \mathrm{~mm}$ which is also statistically significant.

With the use of IZC implants in the posterior region and miniimplants in the anterior region, distalization of the complete maxillary arch and intrusion of the maxillary anterior teeth were adequately achieved which is clinically and statistically significant, by which mild skeletal discrepancy could be corrected nonsurgically.

\section{Limitations}

There are hardly any complications associated with the use of IZC implants. Ideally, fully erupted third molars are to be removed to create a space and aid in distalization process. For unerupted third molars placed below the cemento-enamel junction of the second molars in young individuals, distalization is possible without their extraction. The most common complications associated with bone screws are gingival overgrowth on the screw and early loosening of the screw. The incidence of gingival overgrowth is less with screws with larger heads. In case of early loosening, the implants can be replaced at different sites.

\section{Conclusion}

Treatment of class II malocclusion with mild skeletal discrepancy and gummy smile can be treated nonsurgically and with a nonextraction approach effectively with the use of IZC implants and anterior mini-implants by distalizing the entire maxillary arch and intrusion of the maxillary anterior segment.

\section{References}

1. Melsen B. Mini-implants: where are we? J Clin Orthod 2005;39(9):539. PMID: 16244412.

2. Papadopolous MA. Non-compliance approaches for management of class II malocclusion. In: Current principles and techniques. Edinburg: Elsevier, Mosby; 2006.

3. Gainsforth BL, Higley LB. A study of orthodontic anchorage possibilities in basal bone. Am J Orthod Oral Surg 1945;31(8):406-417. DOI: 10.1016/0096-6347(45)90025-1.

4. Fayed MM, Pazera P, Katsaros C. Optimal sites for orthodontic miniimplant placement assessed by cone beam computed tomography. Angle Orthod 2010;80(5):939-951. DOI: 10.2319/121009-709.1.

5. Chen YJ, Kao CT, Huang TH. Evaluation of ten extra-alveolar temporary anchorage device insertion sites by cone beam volumetric computer tomography: a pilot study. J Dent Sci 2010;5(1):21-29. DOI: 10.1016/ S1991-7902(10)60004-9.

6. Ghosh A. Infra-zygomatic crest and buccal shelf-orthodontic bone screws: a leap ahead of micro-implants-clinical perspectives. J Indian Orthod Soc 2018;52(4_suppl2):127-141. DOI: 10.4103/jios. jios_229_18.

7. Hsu E, Lin JSY, Yeh HY, et al. Comparison of the failure rate for infrazygomatic bone screws placed in movable mucosa or attached gingiva. Int J Orthod Implantol 2017;47.

8. Lin JJ, Roberts WE. Guided infra-zygomatic screws: reliable maxillary arch retraction. Int J Orthodont Implantol 2017;46.

9. Lee JY, Chae HS, Kim YH. Total arch distalization and control of transverse discrepancy with TADs. In: Temporary anchorage devices in clinical orthodontics. 2020. p. 213-221.

10. Lee KJ, Kim SJ. Advanced biomechanics for total arch movement and non-surgical treatment for hyperdivergent faces. Semin Orthod 2018;24(1):83-94. DOI: 10.1053/j.sodo.2018.01.008. 
11. Mohamed MF. Treatment of gummy smile with maxillary anterior teeth intrusion using miniscrews.

12. Shetty SK, Mahesh Kumar Y, Vijayan LG, et al. Advanced methods of total arch distalization in orthodontics: a literature review. Int J Sci Res 2018;7:426. DOI: 10.21275/ART20196070.

13. Singh G, Gupta N, Goyal V, et al. En masse distalisation of maxillary arch using TADs (IZC); passive self-ligating appliance v/s clear aligner - a comparative cephalometric study. J Contemp Orthod 2019;3(3):11-17.

14. Wang $\mathrm{CH}, \mathrm{Wu} \mathrm{JH}$, Lee $\mathrm{KT}$, et al. Mechanical strength of orthodontic infrazygomatic mini-implants. Odontology 2011;99(1):98-100. DOI: 10.1007/s10266-010-0142-3.

15. Creekmore TD. The possibility of skeletal anchorage. J Clin Orthod 1983;17(4):266-269. PMID: 6574142.

16. Doruk C, Çankaya ÖS, Güvenç is. Non-extraction treatment of skeletal class II adult patient with total maxillary arch distalization. Turkish J Orthod 2015;28(3):122-128. DOI: 10.5152/TurkJOrthod. 2016.15-00021R1.

17. Sugawara J, Daimaruya T, Umemori M, et al. Distal movement of mandibular molars in adult patients with the skeletal anchorage system. Am J Orthod Dentofacial Orthop 2004;125(2):130-138. DOI: 10.1016/j.ajodo.2003.02.003.

18. Sung EH, Kim SJ, Chun YS, et al. Distalization pattern of whole maxillary dentition according to force application points. Korean J Orthod 2015;45(1):20-28. DOI: 10.4041/kjod.2015.45.1.20.

19. Lin JC, Yeh C, Liou EJ, et al. Treatment of skeletal-origin gummy smiles with miniscrew anchorage. J Clin Orthod 2008;42(5):285. PMID: 18771123.

20. de Almeida MR. The biomechanics of extra-alveolar TADs in orthodontics. In:Temporary anchorage devices in clinical orthodontics. 2020. p. 445-454.

21. Yoshida N, Koga Y, Mimaki N, et al. In vivo determination of the centres of resistance of maxillary anterior teeth subjected to retraction forces. Eur J Orthod 2001;23(5):529-534. DOI: 10.1093/ejo/23.5.529.
22. Upadhyay M, Nagaraj K, Yadav S, et al. Mini-implants for en masse intrusion of maxillary anterior teeth in a severe Class II division 2 malocclusion. J Orthod 2008;35(2):79-89. DOI: 10.1179/ 146531207225022491.

23. Chung KR, Choo H, Kim SH, et al. Timely relocation of mini-implants for uninterrupted full-arch distalization. Am J Orthod Dentofacial Orthop 2010;138(6):839-849. DOI: 10.1016/j.ajodo.2009.02.035.

24. De Almeida MR, De Almeida RR, Nanda R. Biomechanics of extraalveolar mini-implant use in the infrazygomatic crest area for asymmetrical correction of class II subdivision malocclusion. APOS Trends Orthod 2018;8(2):110-118. DOI: 10.4103/apos.apos_25_18.

25. Bechtold TE, Kim JW, Choi TH, et al. Distalization pattern of the maxillary arch depending on the number of orthodontic miniscrews. Angle Orthod 2013;83(2):266-273. DOI: 10.2319/032212-123.1.

26. Sanap S, Swami V, Patil A, et al. Evaluation of efficacy of various implants in maxillary arch distalization - a finite element analysis. Orthod J Nepal 2020;10(1):44-54. DOI: 10.3126/ojn.v10i1.31005.

27. Liou EJ, Chen PH, Wang YC, et al. A computed tomographic image study on the thickness of the infrazygomatic crest of the maxilla and its clinical implications for miniscrew insertion. Am J Orthod Dentofacial Orthop 2007;131(3):352-356. DOI: 10.1016/j. ajodo.2005.04.044.

28. Kim HJ, Yun HS, Park HD, et al. Soft-tissue and cortical-bone thickness at orthodontic implant sites. Am J Orthod Dentofacial Orthop 2006;130(2):177-182. DOI: 10.1016/j.ajodo.2004.12.024.

29. Kuroda S, Yamada K, Deguchi T, et al. Root proximity is a major factor for screw failure in orthodontic anchorage. Am J Orthod Dentofacial Orthop 2007;131(4):S68-S73. DOI: 10.1016/j.ajodo.2006.06.017.

30. Saxena R, Kumar PS, Upadhyay M, et al. A clinical evaluation of orthodontic mini-implants as intraoral anchorage for the intrusion of maxillary anterior teeth. World J Orthod 2010;11(4):346. PMID: 21491000.

31. Almeida MR. Biomechanics of extra-alveolar mini-implants. Dental Press J Orthod 2019;24(4):93-109. DOI: 10.1590/2177-6709.24.4.093109.sar. 